\title{
El legado pedagógico de Dorothy Lee y Paulo Freire
}

Azril Bacal Roij

bazril@gmail.com

Departamento de Sociología, Universidad de Uppsala

CIPAE-Puebla, México

Red Universidad y Compromiso Social de Sevilla, España

IRIPAZ-Guatemala

Recibido: 24 de agosto del 2020

Aceptado: 28 de septiembre del 2020

\section{Resumen}

El propósito del presente artículo es la incorporación de las valiosas contribuciones de Dorothy Lee, Paulo Freire y otros autores al debate actual sobre el aprendizaje activo. Al respecto, prestamos algunas ideas a partir de la tradición humanista en la antropología cultural de Dorothy Lee: sobre la valorización del yo, el aprendizaje de otras culturas, la comunidad como anclaje de nuestra identidad y la importancia de valores culturales tales como libertad y autonomía para el proceso de enseñanza /aprendizaje. También se extraen algunas contribuciones clave de Paulo Freire en cuanto a la educación transformadora y su relación con los movimientos sociales, la intervención espacio-temporal, la “concientización”, el pensamiento crítico, la esperanza, la educación ciudadana, así como el empoderamiento que resulta de la acción cultural y la transformación social. Estos valores fueron incorporados en un primer acercamiento al aprendizaje activo. El autor argumenta que las ideas de Dorothy Lee y de Paulo Freire desafían el paso del tiempo y siguen iluminando el quehacer en los campos del aprendizaje activo, antropología humanista, educación ciudadana, diálogo intercultural y la investigación-acción participativa. El legado de Dorothy Lee y de Paulo Freire es considerado como una contribución valiosa a los recursos pedagógicos y de investigación para contrarrestar las amenazas que nublan el horizonte de la democracia del siglo XXI.

Palabras clave: guerra cultural, libertad, yo, autonomía, aprendizaje activo, democracia, educación ciudadana, diálogo intercultural. 


\section{The pedagogical legacy of Dorothy Lee and Paulo Freire}

The aim with this conceptual article is to add the valuable contributions of Dorothy Lee, Paulo Freire, and selected authors, to the contemporary debate on active learning, participatory action-research, and intercultural dialogue. In this respect, insights on "valuing the self," learning from other cultures, community as a socio-cultural anchor for identity, and the significance of cultural values such as freedom and autonomy, for the teaching/learning process, are draw from Dorothy Lee's humanist tradition of cultural anthropology. For similar reasons, several key pedagogical contributions are drawn from Paulo Freire concerning transformative education, regarded as closely related to: social movement, spatial-temporal intervention, awareness-raising, critical thinking, hope, citizen education, and empowerment, related to cultural action and social transformation. Such values were actually incorporated in the early approaches to active learning. I contend that the insights of Dorothy Lee and Paulo Freire defy the passage of time, and continue to shed light on the potential roles played by active learning, humanistic anthropology, citizenship education, intercultural dialogue, and participatory action-research. The legacy of Dorothy Lee and Paulo Freire, among other authors, is regarded as a valuable contribution to the available arsenal of pedagogical and research resources, to counteract the ongoing cultural warfare and resulting threats to democracy in the $21^{\text {st }}$ century.

Keywords: Cultural Warfare, Freedom, Self, Autonomy, Active Learning, Democracy, Citizen Education, Intercultural Dialogue.

\section{La Crisis del Siglo XXI}

La humanidad enfrenta un contexto cargado de retos y desafíos globales en varios frentes: pandemias zoonóticas, una sexta extinción masiva de especies animales, una grave crisis social socio-política, económica, cultural, racial y étnica. Estos problemas están estrechamente relacionados entre sí. Beck (1999) discutió estos desafíos en términos de "riesgo global" y de una "crisis mundial", manifiesta en las crecientes desigualdades globales, devastación ambiental, calentamiento global y otros problemas relacionados. Estas situaciones se complican con las condiciones generadas por la pandemia del SARS-CoV-2 
(COVID-19), el aumento dramático de la migración internacional, un mal manejo de la catástrofe humanitaria de refugiados, acompañada de nuevas olas de xenofobia, intolerancia cultural, gobiernos autoritarios, nacional-populismo, neofascismo, violencia globalizada, crimen y corrupción, amenazas nucleares, terrorismo, inseguridad y el debilitamiento de las democracias sociales y liberales.

Las ciencias sociales nos enseñan que nuestra forma particular de "leer" el contexto se refleja en la manera en que nos comportamos como individuos y como grupos. En otras palabras, las definiciones de nuestras realidades compartidas se aprenden a través de la socialización y de la aculturación. Una vez que estas definiciones son impresas e internalizadas como creencias personales, valores y mitos, ellas enmarcan y condicionan nuestro comportamiento personal y acción colectiva. Por esta razón, la educación, los medios de comunicación y la cultura tienen un rol preponderante y estratégico en el deterioro y/o la mejora de la crisis mundial del siglo XXI. Por lo anterior, se entiende por qué la educación y los medios colectivos de comunicación son un campo de disputa acalorada en la guerra cultural entre las agencias públicas y privadas de control social y los movimientos sociales que luchan por la libertad, justicia y democracia.

La creación de las Naciones Unidas, la UNESCO y la Declaración Universal de los Derechos Humanos de 1948 fueron esfuerzos de la comunidad internacional para superar los traumas del siglo veinte. Tales esfuerzos reflejan la confianza depositada en la ciencia, la educación y a la cultura, para reconstruir el mundo a partir de las cenizas dejadas por la Segunda Guerra Mundial. Debido a que las causas profundas de la guerra y la violencia yacen en la mente humana, se invirtió mucha esperanza, creatividad, investigación, esfuerzos y recursos materiales, para mejorar las condiciones socio-educativas para fortalecer la democracia, el estado de derecho, la justicia, la paz y el desarrollo mundial.

¿Qué podemos decir de la situación actual? El mundo civilizado se ha visto forzado a recurrir, una vez más, a la educación, la ciencia y la cultura, para identificar, mitigar y superar los múltiples aspectos de la crisis que hoy enfrentamos. Este contexto le otorga al presente documento un enfoque basado en las estrategias del aprendizaje activo en educación superior. Al respecto, surgen algunas preguntas iniciales: ¿hasta qué punto necesitamos hoy 
de las teorías vigentes, las nuevas políticas y las estrategias para promover liderazgos y adoptar medidas correctivas para abordar de manera efectiva los varios frentes de la crisis global? Estos ámbitos ofrecen un marco para entender y explicar el estado del mundo al borde del desastre.

Si bien no podemos culpar a las empresas productoras de conocimiento de la crisis, sí podemos argumentar con fundamentos razonables que algo de valor se perdió a raíz de los proyectos educativos producidos por la modernidad y la posmodernidad. Sostengo al respecto que algunas distorsiones en la empresa del conocimiento pueden atribuirse al proyecto de capital humano de la educación. Me refiero concretamente al proyecto globalizante neoliberal del fundamentalismo de mercado, aproximadamente desde mediados de los años sesenta (De Vincenzi, 1970). Por lo anterior, propongo colocar los valores formativos de la libertad, el "yo", la autonomía, la comunidad y la ciudadanía en el centro del eje pedagógico en el diseño curricular y la praxis educativa. Esta tarea implica un enfoque transformador del aprendizaje activo y un tipo de investigación encaminado a la resolución de problemas para el bien común, actualmente bajo asedio.

\section{Asedio al Aprendizaje Activo}

En los últimos años, el aprendizaje activo ha recibido una considerable atención... de manera general se define como cualquier método instruccional que involucra a los estudiantes en el proceso de aprendizaje. En resumen, el aprendizaje activo requiere que los estudiantes realicen actividades de aprendizaje significativo.

Michael Price (2004, p.1)

Lo que conocemos como "aprendizaje activo" constituye una empresa y un logo comercial bien establecidos, como resultado de valiosas aportaciones de filósofos, psicólogos, pedagogos, investigadores y otros profesionales dedicados a una amplia gama de disciplinas y campos de estudio. Algunos autores relacionados con el aprendizaje activo son: Freire (1970), Revans (1982), Barnes (1989), Glenda (1996), Bandura (2001), Prince (2004), Brookfield (2005), Weltman (2007), Bonwell y Eison (1991), Michael (2006), Martyn (2007) 
y McKeachie y Sviniki (2006). Más allá de las diversas concepciones de la enseñanza y de las buenas intenciones de los autores mencionados, se observa un cambio en las estrategias de aprendizaje activo durante las últimas décadas, debido a la hegemonía del mercantilismo globalizado y a la privatización de la educación vista como negocio. Este nuevo proceso de educación como mercancía son evidentes en: la creciente dependencia de las directrices de mercado, la evaluación de la educación en términos reduccionistas del costo-beneficio, privatización in crescendo, estímulo a las actitudes de competencia, el desarrollo tecnológico y adopción del enfoque instruccional de la educación.

Desde otro ángulo de análisis, Castells (1996) afirma que las estrategias de aprendizaje activo cambian en la actualidad como respuesta a las demandas de las redes emergentes de información, de conocimiento y a las sociedades cada vez más complejas; que van a la par de esfuerzos regionales y globales para estandarizar los planes de estudio y la praxis en educación superior. Bajo las crecientes presiones ejercidas por estas nuevas demandas globales, el enfoque de las estrategias de enseñanza activa pareciera reducirse a la búsqueda de métodos más efectivos y técnicas instruccionales para motivar y despertar a los alumnos de su supuesto letargo intelectual. No obstante, una revisión a la literatura nos revela la existencia de una vasta investigación y amplia evidencia de que las diferentes disciplinas e instituciones de educación superior se adhieren en gran medida a las premisas de la educación activa. Asimismo, en la mayoría de los centros de educación superior del mundo industrializado se encuentra una variedad de métodos y técnicas disponibles para apoyar la enseñanza activa.

En otras palabras, la teoría, la investigación, los métodos y técnicas de aprendizaje activo no brillan por su ausencia. Además, la investigación confirma que los métodos y técnicas de aprendizaje activo funcionan de manera eficaz. En consecuencia, si la teoría y la investigación nos dicen que por lo general se trabaja bien y que todo está bajo control en el ámbito del aprendizaje activo, ¿qué podríamos añadir, en forma constructiva, al proyecto del aprendizaje activo? Una respuesta preliminar a esta pregunta la encontramos no en la confianza depositada en el más reciente aparato tecnológico educativo, ni en el enfoque 
científico sobre el proceso de aprendizaje, sino en algún locus en las percepciones y lecciones sobre educación formativa asociadas con la tradición humanista y el pensamiento crítico.

Un propósito con el presente artículo es plantear una serie de cuestiones y preguntas, desde una perspectiva democrática sobre la educación ciudadana, la diversidad, la inclusión social, la justicia educativa y la democracia del conocimiento. Esta perspectiva va más allá del enfoque liberal convencional de la igualdad de oportunidades educativas. En otras palabras, se tiene en mente un proyecto educativo alternativo que apoya la igualdad en el acceso a la educación superior de calidad para tod@s los estudiantes sin importar su nacionalidad, clase, género, raza, origen étnico, preferencia sexual, ni otros criterios de exclusión social.

Un principio sociológico bien conocido de las profecías autocumplidas afirma que "aquello que se define como real llega a tener consecuencias reales". En consecuencia, si creemos que los estudiantes carecen de motivación propia para aprender, nuestros esfuerzos educativos se centrarán en motivarlos a través de métodos y técnicas externas. Por otra parte, si consideramos que los estudiantes están intrínsecamente motivados para aprender de manera activa, nuestros esfuerzos para mejorar su aprendizaje se centrarán en la atención activa que nosotros como profesores les proporcionamos; así como en nuestra responsabilidad para rediseñar las instituciones existentes de educación para apoyar de manera efectiva la curiosidad innata para aprender que cargan en su mochila todos los estudiantes. A continuación, se presenta una perspectiva de valor sobre la libertad, el "yo", y la autodeterminación; aplicada al ámbito del aprendizaje activo.

\section{Una perspectiva de valor sobre el yo, la autonomía y la autodeterminación}

La ciencia puede ser racional únicamente si tiene premisas de valor explícitas. Se necesitan premisas de valor para poder interpretar los hechos. No hay respuestas, sin preguntas. No hay preguntas, sino a partir de puntos de vista.

Gunnar Myrdal (1973)

Revista RedCA octubre 2020 - enero 2021 ISSN: 2594-2824 Vol. 3 Núm. 8 
Las siguientes líneas presentan mis reflexiones sobre el rol estratégico que los valores concebidos por Dorothy Lee y Paulo Freire juegan en las esferas de la investigación transformadora y la educación. Estos autores ocupan un lugar importante en la lista de los grandes educadores del siglo XX - y aunque ambos valoraban el diálogo en su praxis pedagógica y tenían un gran respeto por la autonomía y la libertad de cada estudiante, también difieren en algunos aspectos, como se verá a continuación. Dorothy Lee intentaba, desde un estilo de enseñanza único y comprometido, ir más allá del etnocentrismo lingüístico, cultural, perceptual y cognitivo de sus estudiantes, invitándolos a aprender valores de "culturas remotas".

Freire nos brinda una forma de enseñanza-aprendizaje para sensibilizar a los alumnos, haciendo uso de estrategias tales como: la "pedagogía de la pregunta", la dialéctica y el diálogo. El uso de la dialéctica es una manera de conducir a los alumnos a reflexionar sobre su propio pensar, así como respecto a la dinámica y las contradicciones inherentes a su cultura y sociedad. El diálogo alude a la escucha activa de los demás, como medio para promover la empatía, invitando a los estudiantes a "ponerse los zapatos del otro", una manera de hablar que se refiere al esfuerzo por entender mejor los puntos de vista del alter. Como no lo sabemos todo, y el otro tampoco lo sabe, Freire nos invita a aprender la una del otro. Desde esta visión, todos somos co-aprendices y co-investigadores en el juego educativo. ¿Cómo transmitir el sentido de urgencia para re-incorporar la dimensión formativa originaria del aprendizaje activo, en la educación superior y en la investigación comprometida con el bien común?

\section{El contexto educativo: una invitación a "leer" y transformar el mundo}

De acuerdo con István Mészáros (2004, citado en Bacal, 2014, p. 69), el proceso de enseñanza y aprendizaje se ve facilitado o bloqueado, simultáneamente, por el contexto situacional y los actores clave en el juego educativo. El papel del docente es, por naturaleza, dual; es decir, facilita u obstaculiza de manera activa el proceso de aprendizaje del alumn@. Además, el contexto del aprendizaje activo implica más que el mero aspecto espacial. El contexto educativo se encuentra enmarcado por el tiempo y el aprendizaje activo se produce dentro de límites espacio-temporales. De una y otra manera, las nuevas olas de reforma 
educativa modifican estos tiempos y espacios socio-educativos ya construidos. Asimismo, estos límites contextuales e institucionales pueden cambiar mediante la intervención de los actores educativos, que participan de manera activa en el proceso de enseñanza/aprendizaje. De lo anterior se desprende que debido a nuestros múltiples roles, en calidad de profesores, investigadores, con capacidad de decidir, como planificadores y administradores de la educación, somos agentes clave en el contexto aludido. En otras palabras, no somos meros espectadores, mirando desapasionadamente, la escena educativa - y sí somos, más bien, jugadores clave, cargados con demasiada ventaja en la definición de las reglas y rutinas del juego educativo. En este sentido, ¿qué tan listos y dispuestos estamos para examinar las consecuencias de nuestros actos en ese escenario?

Para terminar con mis reflexiones acerca del contexto de la enseñanza/aprendizaje, me pregunto si las escuelas e instituciones de educación superior están en condiciones de ofrecer de manera activa a sus estudiantes un espacio formativo de aprendizaje, para que desarrollen su capacidad de pensamiento crítico y, además, invitarlos a participar como ciudadanos-estudiantes en el gobierno compartido de sus centros de estudio? Si la respuesta es afirmativa, se espera que la educación en la etapa inicial de formación en la teoría y práctica de los valores democráticos logren, posteriormente, fomentar su compromiso como ciudadanos en la sociedad, la cultura y la comunidad. Esta pregunta me lleva a introducir brevemente a Dorothy Lee, en el marco de la tradición humanista y existencial de la antropología cultural relacionada con la educación.

\section{Una breve introducción a Dorothy Lee}

Dorothy Lee compartía su interés e involucramiento en el campo de la educación con Margaret Mead y otros antropólogos. Fue la última de nueve hijos de una familia evangélica griega. Creció en un ambiente mayormente ortodoxo griego en la Estambul musulmana (antes Constantinopla) en 1905. La siguiente información es extraída de los trabajos de Jeffrey Ehrenreich, antropólogo que descubrió a Dorothy Lee durante sus estudios de postgrado con Edmund Carpenter. Es mejor transmitir el significado del trabajo de Dorothy Lee con sus propias palabras:

Revista RedCA octubre 2020 - enero 2021 ISSN: 2594-2824 Vol. 3 Núm. 8 
El impulso original para adentrarme en la dimensión de la cultura vino de mi esposo, Otis Lee (filósofo), para quien toda experiencia y comportamiento contiene un contenido filosófico y toda realidad tiene valor. Mi búsqueda a partir de la codificación cultural de la realidad experimentada, de las implicaciones valorativas y conceptuales del lenguaje, y otros aspectos de la cultura, vinieron de un intento por encontrar respuesta a sus inquietantes preguntas. En años recientes, me han preocupado cuestiones en torno a la libertad, autonomía individual, responsabilidad, creatividad y el "yo". (1986, pp. v-xii)

Según Ehrenreich, Dorothy Lee estaba profundamente preocupada por la siguiente cuestión: "¿de qué manera se conectan la autonomía y la libertad individual con la comunidad y la sociedad?" (1986, p. 176). En contraste con la visión neoliberal de la libertad, tanto Dorothy Lee como Paulo Freire compartían la concepción de la libertad y la autonomía como socialmente engarzada en la comunidad. Para ilustrar este punto, en los años sesenta, Dorotthy Lee enseñaba en los plantones de la Universidad de California en Berkeley y donaba sus honorarios como oradora al Movimiento por la Libertad de Expresión. Se le invita al lector a reflexionar, acerca de y hasta qué punto, la cultura imperante en el contexto laboral valora y apoya de manera activa la autonomía de sus estudiantes, como integrantes de la comunidad de aprendizaje, con un papel distinto. Ehrenreich describe la visión de Dorothy Lee sobre la autonomía:

A través del análisis comparativo de numerosas culturas, occidentales y remotas, Lee sugería que para que el individuo pudiera desarrollar su autonomía, era esencial que la comunidad realmente valorara el "yo". (1986, pp. v-vi)

De acuerdo con Dorothy Lee, el valor que las distintas culturas otorgan a la libertad y a la integridad del joven como estudiante, varía dentro de sus límites culturales. Desde una perspectiva comparativa, se encuentran culturas que valoran el yo (Lee, 1986) y la autonomía de los estudiantes (Freire, 1997), y también hay "culturas en contra del hombre" (Henry, 1963). De manera ilustrativa, Jules Henry estudió las culturas ue valoran y ejercen una praxis 
educativa de obediencia, disciplina, control social, conformismo y "asesinato del alma", epitomizada mas no restringida por la pedagogía Nazi (Schatzman, 1973).

En el espacio compartido entre estudiante y alumno que concebido por Dorothy Lee, los estudiantes son considerados y tratados como "curiosos por naturaleza" y activamente involucrados en la exploración, aprendizaje y desarrollo de una relación íntima con sus "universos relevantes". Expresado de otra manera, los estudiantes no necesitan de motivación externa en términos de recompensas y/o castigos, como recomiendan los educadores de orientación conductista. Lee era una acérrima crítica de B.F. Skinner, colega suyo en la Universidad de Harvard, por su manifiesta falta de respeto hacia sus estudiantes, quienes eran continuamente tratados como ratas de laboratorio. Las dudas respecto del mal definido problema de la pasividad de los estudiantes fueron extendidas por Dorothy Lee a las correspondientes medidas correctivas, construidas en base a equivocadas suposiciones sobre la "necesidad" de técnicas de motivación y refuerzo externo. Este sería el caso, incluso si dichas medidas correctivas por parte de quienes toman decisiones en la educación son bien intencionadas, al pretender transformar estudiantes apáticos en estudiantes activos. Desde nuestra visión, el contexto educativo está compuesto por instituciones de educación superior y se refiere particularmente al locus "espacio-temporal" mediado por la calidad de la relación humana entre el profesor y el alumno.

Cuando tuvimos nuestra primera clase, Dorothy Lee nos pidió que imaginemos y decidamos, si nos quedamos o no en el aula en caso de que el mundo se acabara repentinamente. De esa manera ella nos confronta con una elección existencial: o quedarnos con ella en el aula o marcharnos. En nuestros días, plagados de crisis existencial, ausencia de sentido de vida y desesperanza, la pregunta provocadora que Dorothy Lee, hecha en la primera clase, infundió mi vida desde entonces con el deseo de búsqueda del sentido de las cosas, que transformó mi vida personal y estilo de enseñanza con consecuencias que duran hasta el día de hoy. Dorothy Lee discutió el tema de la ausencia de sentido en la educación en términos de una crisis existencial reflejada, por ejemplo, en el consumismo masivo y la cultura de la droga. Además, atribuía dicha crisis a la notable desaparición del sentido de comunidad en el mundo contemporáneo. Esta autora no es la única que encuentra vínculos 
orgánicos entre el proyecto educativo de la modernidad, el capitalismo, la alienación y a la anomia en la sociedad contemporánea. La visión de Dorothy Lee sobre la urgente necesidad de reconstruir la comunidad es compartida con Martin Buber (Marin, 2016, p. 56) y los epidemiólogos Wilkinson y Prickett (2013).

Considerando lo anterior, ¿podemos concebir las instituciones contemporáneas de aprendizaje activo en la educación superior como comunidades de aprendizaje que reconocen, respetan y apoyan la libertad, el "yo," la autonomía, el desarrollo personal y la educación ciudadana de sus estudiantes? Paulo Freire, como se verá a continuación, ofrece una respuesta a dicho interrogante, con una visión comprometida de la educación y praxis educacional.

\section{Una breve introducción a Paulo Freire}

Desde una perspectiva de la pedagogía humanista y crítica, en esta sección se hace una breve presentación de Paulo Freire y sus contribuciones al aprendizaje activo, basado en libertad, autonomía, diálogo, dialéctica y educación transformadora, encaminado a la acción cultural y al cambio social.

Mi encuentro con el trabajo seminal de Paulo Freire se remonta a su trabajo sobre extensión versus la comunicación (Freire, 1973). Freire describe a la extensión agrícola como un tipo "monológico" de comunicación persuasiva y opresiva, definida en contraste con el tipo "dialógico" (liberador) de la comunicación. Paulo Freire fue influído por el enfoque socrático (mayéutico) del proceso de enseñanza/aprendizaje y en particular por el trabajo filosófico de Martin Buber (1955).

Buber fue un filósofo social y político que realizó una distinción teórica entre dos modos de interacción y comunicación. Aplicados al campo de la educación superior, estos dos modos corresponden a dos caminos de enseñanza/aprendizaje: 1) La modalidad "Yo ello", en la que el "otro" (el profesor) ve al estudiante como un "ello", un objeto al que se le debe enseñar y moldear a gusto, y 2) La modalidad "Yo - Tú", basada en el respeto, el cuidado

y la apreciación mutua. Aquí, la enseñanza se entrelaza con el aprendizaje a través del diálogo 
y donde las funciones del profesor y los estudiantes se combinan, permitiéndoles aprender el uno del otro, en calidad de co-profesores/aprendices.

Mi argumento sobre la pertinencia del diálogo en la enseñanza superior es el siguiente: los entornos de aprendizaje con primacía de la modalidad de enseñanza "yo-ello" afectan el aprendizaje activo, y viceversa. En la modalidad de enseñanza "yo-tú", el aprendizaje activo se desarrolla. Aunque se reconoce que Paulo Freire fue uno de los pioneros de la teoría del aprendizaje activo, algunos de los principios humanistas y críticos de la educación asociados a su obra actualmente se han perdido o ignorado como resultado de la prisa y el ruido del rápido ritmo del mundo globalizado en que vivimos.

En este marco de pensamiento, ¿qué tan válida es la suposición ampliamente difundida en los círculos conductistas, postulando que los estudiantes son pasivos y, en consecuencia, necesitan motivación y refuerzo externos? Sobre esto, se discute una visión alternativa que sostiene que la curiosidad de los estudiantes en los primeros años por aprender y por seguir sus intereses, por lo general se ve desdeñada en nuestras rutinas y formas de organizar y gestionar el proceso de aprendizaje. Esto nos recuerda la historia de un hombre que, sentado sobre los hombros de otro, dice "haría todo por ti, excepto bajarme de tus hombros".

En este documento, relacionado con el locus interpersonal, se presenta una perspectiva concerniente con la libertad, la autonomía y la autodeterminación de los estudiantes, como medio para referirse a la interacción entre el profesor y el estudiante en el marco conceptual y praxis del aprendizaje activo. Para ello, me baso en el trabajo seminal de Moacir Gadotti, un destacado pedagogo contemporáneo cuyo trabajo se relaciona estrechamente con Paulo Freire. Este autor recuerda la triada concebida por Rousseau en el juego educativo: 1) "Yo" (el aprendiz activo), 2) "otras personas" (profesores, padres) y 3) "cosas" (en alusión a los rasgos circundantes de la naturaleza y a los artefactos culturales creados por el hombre) (Gadotti, 2000, p. 82). El papel de los "otros (educativos) significativos" como profesores administradores de la educación conlleva un papel doble para facilitar u obstaculizar el proceso de aprendizaje innatamente activo y exploratorio de los estudiantes. 
Las "cosas" adquieren a su vez significados instrumentales y existenciales a través de la interacción humana de los estudiantes con otras personas importantes; una situación interpersonal en la que ambos actores educativos se enmarcan en el juego de aprendizaje para crecer en una comunidad. Las otras personas significativas es un término que se refiere a las personas importantes de nuestro entorno cotidiano, quienes transmiten los significados y nombres culturales vinculados al mundo de las cosas naturales y los artefactos humanos. Este proceso interpersonal de transferencia de conocimientos e información significativa se filtra en todo momento por medio de los valores culturales particulares, el lenguaje y los estilos personales de enseñanza de profesores y otras personas significativas.

El contexto de enseñanza/aprendizaje se refiere al mundo, el aula, y el locus espaciotemporal educativo, a los que Dorothy Lee llamaba "universos relevantes". En otras palabras, los estudiantes recorren su viaje educativo delimitado por un entorno simbólico, poblado por una comunidad de personas significativas, asentado en lugares y tiempos significativos. Se desprende, de este punto de vista que, si el contexto concreto de aprendizaje se experimenta como irrelevante, el proceso de aprendizaje activo se ve afectado; por lo que es probable que los estudiantes consideren que la educación superior sea igualmente vista como carente de sentido (Majfud, 2017). Freire consideraba que el contexto es problemático, explotador, opresivo, alienado y alienante; por lo que el alumno/estudiante debía "leerlo" críticamente. Se esperaba que al sensibilizar a los alumnos/estudiantes como ciudadanos, se les empodera e involucra en la acción cultural para cambiar sus características problemáticas. Desde sus respectivas miradas antropológicas y pedagógicas, Dorothy Lee y Paulo Freire, consideraban la totalidad de la vida humana como un contexto de aprendizaje; es decir, como un terreno fértil para involucrar a los estudiantes en el proceso de aprendizaje activo.

Desde su visión, la apatía de los estudiantes corresponde a un diagnóstico erróneo, fundamentado, a su vez, en una premisa equivocada sobre la pasividad de los alumnos. Además, Dorothy Lee criticaba la visión de moda que afirmaba que los estudiantes necesitaban motivación externa y consideraba que el sistema de evaluación era signo de la falta de respeto, así como de una "degradación” del valor de la integridad personal de los estudiantes. Dorothy Lee consideraba que los estudiantes están intrínsecamente motivados y 
autodirigidos por sus valores e intereses, y que su aparente apatía o pasividad era resultado de un programa y de un contexto educativo sin sentido e irrelevante. Paul Goodman (1960) capturó la visión de la banalidad educativa ya mencionada al señalar las condiciones absurdas de crecer en una sociedad contemporánea alienada.

La influencia transformadora de Paulo Freire en toda mi vida profesoral se ilustra brevemente en la siguiente sección con algunos estudios de casos. Esta sección termina con una pregunta: ¿podemos imaginar una alternativa de aprendizaje activo en las instituciones de educación superior contemporáneas, concebidas como comunidades transculturales bajo los lineamientos del modo "Yo-Tú" de enseñanza/ aprendizaje?

Una manera de ilustrar la manera en que podría funcionar esta alternativa de aprendizaje activo, en contraste con el rápido ritmo de la vida moderna es la lectura lenta y un ritmo más calmado de aprendizaje activo, que se introduce a continuación. Se aplicó una perspectiva "Yo-Tú" sobre el diálogo intercultural y la inclusión social en un estudio de caso de diversidad cultural y desarrollo institucional en la Universidad de Uppsala (Bacal, 1998).

Los hallazgos de este estudio nos dicen que el multiculturalismo no va de la mano con las esperadas líneas igualitarias de la inclusión social en el proceso de desarrollo institucional de esta universidad de gran prestigio. Los puntos de vista pedagógicos y estilos de enseñanza de Dorothy Lee y Paulo Freire también se aplicaron en "Cultura y Etnicidad", un curso diseñado ad hoc cuando invitado a enseñar como Profesor Visitante de Humanidades en el Otterbein College, Ohio (en el otoño de 1995) y cuyas principales características se comentan a continuación.

Este curso se diseñó para proporcionar un enfoque teórico, metodológico y experimental para el estudio de la cultura, la etnicidad y la identidad étnica. La metodología de este curso se basó en la participación y el diálogo, con la incorporación de proyectos individuales, en pequeños grupos y niveles de trabajo y debate en el aula. La diaria lectura y escritura personal se combina con círculos de estudio, que a su vez funcionan como grupos de apoyo entre pares. El formato de las disertaciones se complementa con seminarios y 
discusión grupal. Las películas y los proyectos de campo también enriquecen la experiencia colectiva de aprendizaje. Se intentó relacionar la dinámica de este curso con las actividades del "festival de estudios integradores", que se centró en la Cultura, el Conflicto y la Comunidad, así como con la Conferencia de Derechos Humanos en Octubre de 1995. El tipo y el ritmo de lectura individual adoptado en este curso siguió las exhortaciones de Dorothy Lee, en cuanto a que debía ser muy, muy lento, para habilitar el tiempo suficiente para caminar, por así decirlo, por las páginas, imaginando a nosotros mismos caminando en los paisajes leídos, percibiendo los olores de la naturaleza, "conociendo" los lugares, "escuchando" e incluso "relacionándose" con las personas que presentan los autores en los libros.

A continuación, se invita a los estudiantes a reflexionar y a escribir acerca de sus profundas reacciones y reflexiones, incluyendo emociones, sentimientos y/o el análisis intelectual. Estas respuestas profundamente personales se comparten y debaten posteriormente en el contexto del diálogo grupal en el espacio áulico. La discusión resultante se enriquece con la pluralidad cultural y las diversas perspectivas de sus miembros. De esta manera, el sentido individual de descubrimiento personal se une a la dimensión analítica en un espacio y proceso [B1]de aprendizaje activo.

Las tareas del curso incluían la redacción de un diario personal con los comentarios, reacciones y reflexiones de los estudiantes sobre sus "encuentros personales" con los personajes y lugares que los "conocieron" en las páginas de los libros asignados en el curso. Estas notas se discutían semanalmente con el profesor, que añadía los reportes de lectura del grupo y otras actividades relacionadas con el curso. Se adoptó un enfoque similar en un curso sobre "Aprendizaje de Culturas Remotas". Esta vez el curso se impartió en la institución de Cultura y Estudios Bibliotecarios de la Universidad de Uppsala en 1998, utilizando "Juan el Chamula", el mismo texto anteriormente mencionado, escrito por Ricardo Pozas Arciniegas. Este curso fue diseñado para introducir y "entrar" en el mundo de los indígenas chamula (mayas) en Chiapas, México.

Revista RedCA octubre 2020 - enero 2021 ISSN: 2594-2824 


\section{Notas finales}

A modo de conclusión acerca de las reflexiones sobre el aprendizaje activo en educación superior, se plantean algunas cuestiones por encima de las nociones de moda de la educación de calidad y las competencias del profesorado que figuran en las directrices de Bolonia sobre educación superior. Ello ocurre en el contexto de un retorno en el mundo occidental al autoritarismo, a la ultraderecha, y al populismo nacionalista blanco. Esta pregunta se plantea en los siguientes términos: ¿podemos prever y ayudar a construir un enfoque alternativo de la educación activa en las instituciones contemporáneas de educación superior, en el que el tempo individual de los estudiantes sea reconocido y apoyado por profesores $\mathrm{y}$ administradores de comunidades de aprendizaje? ¿Podría la visión alternativa del aprendizaje activo, esbozada anteriormente, sustituir el actual enfoque individualista, competitivo y lleno de estrés, asociado con el ritmo rápido y la educación superior mercantilizada de nuestros tiempos?

Al tener en cuenta la gran demanda de tiempo y compromiso que requiere el enfoque Yo-Tú del proceso de enseñanza/aprendizaje, es fácil comprender por qué hoy en día se encuentra raramente dicha modalidad en las instituciones de educación superior. No obstante, valdría la pena reflexionar como Prometeo, contra todo pronóstico, sobre el valor de construir un enfoque educativo alternativo de aprendizaje activo y de investigación comprometida con el bien común, que valore y apoye la libertad, el "yo" y la autonomía de los estudiantes. Ciertamente, dicho enfoque suena poco realista en nuestra era de comida rápida, lectura rápida, cursos que duran una semana y falta de interés por el pensamiento crítico y la educación ciudadana, que hoy prevalecen en la mayoría de las instituciones occidentales de educación superior.

Para hacer llegar este punto a mis maestros-estudiantes mexicanos a nivel doctoral, les muestro una entrevista en video con un estudiante que acaba de graduarse del bachillerato en Finlandia, el país con los resultados más altos de PISA en el mundo. La pregunta es la siguiente: “Ahora que te has graduado de la escuela, ¿qué deseas hacer?, ¿qué trabajo te interesa más?” Su respuesta fue: "Quiero ser gerente de Coca-Cola.” ¿Es este el mejor producto de lo que hoy se conoce como educación de calidad? No olvidemos que el término 
"educación de calidad" fue importado del significado original que se le dio a la calidad en el mercado de productos básicos. En tan reducido significado de la educación, se pierde algo valioso en relación con las dimensiones formativas y éticas del aprendizaje activo y la educación superior.

Yo argumento que en las condiciones mundiales y socioeducativas imperantes, la valoración del yo, la autonomía, el diálogo, la educación multidisciplinaria y transcultural, y la educación ciudadana en particular, son urgentemente necesarias para ocupar un lugar central en el eje de los planes de estudio, el diseño institucional y la praxis educativa y de investigación. En estas líneas se encuentra la promesa de una visión alternativa del aprendizaje activo en la educación superior basada en el diálogo, apreciado como un atributo clave del aprendizaje activo enmarcado en la educación interpersonal.

Mis reflexiones y sugerencias para mejorar el aprendizaje activo se conciben no sólo como crítica a rajatabla, sino también como complemento o suplemento a todos los esfuerzos por traer entusiasmo al aprendizaje en el aula, por buscar mejores alternativas que lleven a la sustitución de las formas tradicionales de enseñanza (pasiva) y, por último, para aprender de los sistemas educativos más importantes sobre competencias y aprendizaje de calidad en lectura, matemáticas y ciencias, sin olvidar el valor del estudio de las humanidades.

El aprendizaje activo de calidad también se relaciona con la educación ciudadana formativa. Para que la democracia, si merece su nombre, tenga un futuro viable en el siglo XXI, es necesario que los planificadores y actores clave de las instituciones de enseñanza superior reconozcan, nutran e incorporen en los planes de estudio y en la práctica diaria del aprendizaje activo, los valores de la libertad, el yo, la autonomía, el diálogo, el entendimiento y la apreciación intercultural y la democracia.

En esta época descrita en términos de tecno-barbarismo, Dorothy Lee y Paulo Freire siguen siendo un faro en las aguas turbulentas de la educación superior. Si estuvieran vivos hoy en día, verían con consternación cómo la libertad, el yo y la autonomía de los estudiantes son términos olvidados en el vocabulario estandarizado y la praxis diaria en la educación superior contemporánea. ¿Qué podemos hacer al respecto? Una perspectiva holística sobre 
el aprendizaje activo y la investigación comprometida con el bien común exigiría la incorporación de todos los actores clave que participan en las instituciones de educación y en la investigación para la resolución de problemas. Paulo Freire compartía con Dorothy Lee un enfoque educativo "comunitario" y sociocultural del proceso de enseñanza y aprendizaje. Aún más, Freire concebía, al igual que María Montessori, a la educación como movimiento social; es decir, como una forma colectiva de intervenir en el ámbito socio-educativo, en una visión que incluye a la imaginación humana. Estos autores entendieron el íntimo nexo establecido entre la libertad, el yo y la autonomía de los estudiantes dentro de los límites construidos de sus culturas y sus estructuras sociales.

Las observaciones y reflexiones anteriores nos confrontan con nuestra postura como profesores y administradores en el juego educativo y de investigación, y plantean las siguientes preguntas: ¿estamos preparados para reflexionar críticamente sobre nuestras contribuciones a la reproducción y/o cambio de los problemas educativos que buscamos resolver? En este sentido, cuando la situación así lo requiera, ¿qué tan dispuestos estamos a cambiar personalmente? Y por último, mas no por ello menos importante, ¿hasta qué punto estamos dispuestos a cuestionar y a comprometernos con la tarea de transformar las instituciones de enseñanza superior en las que trabajamos y pagan nuestros salarios? Es cierto que tales preguntas y dilemas existenciales descritos anteriormente son problemáticos, exigentes y dolorosos de tratar, en comparación con la búsqueda de metodologías y técnicas participativas para activar el aprendizaje de los estudiantes. Estas consideraciones apuntan a una pregunta final: ¿estamos dispuestos a comprometernos en la tarea de entablar un diálogo con los estudiantes dentro y fuera del aula, como co-investigadores e individuos únicos?

\section{Referencias bibliográficas}

Achebe, Ch. (2008). Things Fall Apart. New York: Bantam Doubleday Dell Publishing Group Inc.

Bacal, A. (1998). Reflections on Cultural Diversity and Institutional Human Development, en Karin Apelgren och Ann Blückert, Universitetet som kulturell mötesplats: 
Verkligheten - utmaning - möjlighet! UPPSALA UNIVERSITET. Rapportserie från Enheten för utveckling och utvärdering. Rapport nr 15:51-64.

Bacal, A. (1996). Permeando Muitos Projetos, Cap.5, Parte II, en Moacir Gadotti (ed.), Paulo Freire: Uma Bibliografía, Cortes Editora, UNESCO, Instituto Paulo Freire (IPF), São Paulo, Brasil (232-234).

Bandura, A. (2001). Social Cognitive Theory: An Agentic Perspective, in Annual Review of Psychology, Vol. 52:1 (February).

Barnes, D. (1989). Active Learning. Leeds University TVEI Support Project” (p.19).

Beck, U. (1999). World Risk Society. Cambridge: Polity Press.

Bonwell, C.C., and J.A. Eison (1991), Active Learning: Creating Excitement in the Clasroom, ASHEERIC Higher Education Report No.1, George Washington University, Washington, DC. Brookfield, S.D. (2005), Discussion as the way of teaching: Tools and techniques for democratic classrooms (2nd ed.). San Francisco: JosseyBass.

Buber, M. (1955). Between Man and Man, Boston: Beacon Press.

Castells, M. (1998). The Rise of Network Society. Oxford:Blackwell.

Elias, R. (10 July 2014) (www.ted/talks.Rich Elias).

Freire, P. (1973). Extensión o Comunicación: La Concientización en el Medio Rural. México: Ed. Siglo XXI y Tierra Nueva.

Freire, P. (1997). Pedagogia da Autonomia. São Paulo: Editora Paz e Terra.

Freire, P. (1970). Pedagogy of the Oppressed. New York: Herder \& Herder.

Gadotti, M. (2000). Pedagogia da terra: Ecopedagogia e Educacao Sustentável. CLACSO.

Revista RedCA octubre 2020 - enero 2021 ISSN: 2594-2824 Vol. 3 Núm. 8 
Gerhardt, H. (1993). PAULO FREIRE (1921-97), in Prospects: The quarterly review of comparative education. Paris: UNESCO: International Bureau of Education, vol. XXIII, no.3/4.

Goodman, P. (1960). Growing Up Absurd: Problems of Youth in Organized Society. New York: A Vintage Books.

Henry, J. (1963). Culture Against Man. New York:Vintage Books. A Division of Random House.

Jeffries, S. (2017), in The Guardian, Monday 22 May, pp. 1-10.

Lee, D. (1987, 1959), FREEDOM AND CULTURE. Prologue by Jeffrey Ehrenreich. Prospect Heights, Illinois: WAVELAND PRESS, INC. · Lee, D. (1986, 1976). VALUING THE SELF. Epilogue by Jeffrey Ehrenreich. Prospect Heights, Illinois: WAVELAND PRESS, INC.

Majduf, J. (2017). Educación. ¿Hacia dónde Vamos? Rebelión (01 Junio).

McKeachie \& Svinicki (2008). Teaching and Learning in Higher Education. Academy of Management Learning \& Education. Vol. 7, No. 1 (Mar), pp. 139-142.

Marin, L. (2010). Can we save true dialogue in an Age of Mistrust) Uppsala: Dag Hammarskjöld Foundation. Critical Currents no.8 (January).

Martyn, M. (2007). Clickers in the Classroom: An Active Learning Approach, EDUCAUSE Quarterly (EQ). 30 (2).

Mészáros, I. (2004). Education Beyond Capital. III Foro Mundial de Educación. Porto Alegre, RGS, Brasil, in Bacal (2011, op.cit, p.69).

Michael, J. (2006). Where's the evidence that active learning works? Advances in Physiology Education, 30 (4), 223-231. 
Myrdal, G. (1973). At a lecture at Campbell Hall, University of California at Santa Barbara, 2 November.

Pozas Arciniegas, R. (1962). Juan the Chamula. Berkeley, CA., Berkeley University Press.

Prince, M. (2004). Does Active Learning Work? A Review of the Research, J. Engr. Education, 93(3), 223-231. 23

Revans, R.W. (1982). The Origins and Growth of Action Learning. Bromley: Chartwell-Bratt Ltd.

Rodriguez, R. (1982). Hunger of Memory. New York: Bantham Dell, A Division of Random House, Inc.

Schatzman, M. (1973). Soul Murder. New York: Random House.

Smith, D. (1993). Fires in the Mirror: Crown Heights, Brooklyn and Other Identities. New York: Penguin Random House Inc.

Snyder, T. (2017). On Tyranny: Twenty Lessons from the Twentieth Century. London: The Bodley Head.

Vygotsky, L. (1978). Interaction between Learning and Development. From Mind and Society (pp.79-91). Cambridge, MA: Harvard University Press.

Weltman, D. (2007). A Comparison of Traditional and Active Learning Methods: An Empirical Investigation Utilizing a Linear Mixed Model, PhD Thesis, Arlington: The University of Texas.

\section{Notas}

1. Las presentes reflexiones se basan en mi trayectoria y compromiso de toda la vida en el mundo de la enseñanza superior como profesor universitario nómada e investigador en varios países: Perú, México, EE.UU., España y Suecia; de mi trabajo como consultor en la Oficina Regional de Educación de la UNESCO para América Latina y el Caribe 
(OREALC/UNESCO), de enseñar a profesores en México y de la participación en el Foro Mundial de la Educación y en la red "Universidad y Compromiso Social" en Sevilla, España.

5. Bacal, Azril (1994). Del organismo a la identidad: el camino de la psicología a la psicología social (Hacia una epistemología de la autodeterminación). Universidad de Karlstad, Departamento de Ciencias Sociales, Sección de Comunicación. Documento de trabajo 94:1. Este trabajo expresa una visión de un espacio socio-educativo de libertad, al pasar de la noción biológica de organismo en psicología al concepto socio-psicológico de identidad. Cuando la psicología supera su postura sobre los organismos condicionados externamente ("enseñados") y se dirige hacia la psicología social, se considera que los individuos, como los estudiantes, están dotados de atributos y aptitudes humanas únicas para responder, y no solo para reaccionar a los estímulos externos: autorreflexión, libertad, libre albedrío, autonomía y autodeterminación.

6. Basado en una reciente experiencia dramática, cuando Elías sobrevivió al vuelo 1549 de US Airways que se vio obligado a aterrizar en el río Hudson (www.ted/talks.Rich Elias).

7. Publicado por primera vez en 1976, y también en el epílogo escrito en 1988 añadido a su colección de ensayos sobre libertad y cultura (1959). Reimpreso en 1987 (pp.76-180).

8. Goodman, Paul (1960). Growing up Absurd.

9. Un relato parcial de mi experiencia docente, inspirado en Paulo Freire, se transcribe en el capítulo "Permeando muitos Projetos" en Gadotti (op.cit, 1996:232-234). La experiencia de enseñanza/aprendizaje referida abarca un largo período de tiempo, alcance y una variada gama de actividades que se remontan a las tareas asociadas a la capacitación de campesinos y a los Radio-Foros contextualizados en la Reforma Agraria Peruana (1969-1976). Este enfoque de la pedagogía de la sensibilización se aplicaría posteriormente en un curso experimental sobre "Superación de las consecuencias personales del racismo", en la Universidad de California, Santa Bárbara (1979) - elaborado, más tarde, en términos de "descolonización de la identidad étnica" con mexicoamericanos (1994). Esta perspectiva también se aplicó al ámbito de la autogestión, con trabajos sobre "Una estrategia de organización y capacitación participativa para el sector de la autogestión": un estudio de caso 
de investigación-acción en Perú" (1991:121-135), "La Universidad Iberoamericana en el Contexto de la Globalización" (2000:161-218), "Socioanálisis y Concientización en las Ciencias Sociales" (2002), "Espacios de Intervención Socio-Educativa en el Mundo Actual" (2004), "Movimientos Sociales y Educación Transformadora". Mis opiniones sobre la "educación transformadora" inspirada en Freire fueron resumidas en Bacal (2011:65-84). Por último, pero no menos importante, la reciente experiencia en la formación de docentes en varios programas doctorales en México me permitió explorar e involucrar activamente a mis profesores-alumnos en un esfuerzo grupal para recrear las bases de un aprendizaje activo en educación superior dirigido a la construcción de otros mundos diferentes, orientados al bien común.

10. En un curso similar, mis estudiantes mexicanos de posgrado tuvieron el privilegio de conocer personalmente y conversar con Ricardo Pozas, destacado antropólogo de la Universidad de las Américas, Puebla (UDLAP), México en la década de los ochenta. Este enfoque pedagógico también se había probado anteriormente en Perú, donde mis estudiantes se reunieron con el escritor y antropólogo José María Argüedas para discutir sobre Todas las sangres, un libro fundamental sobre su proyecto de un país plurinacional y multicultural.

11. Snyder (2017).

12. Bonwell, Eison y James (1991).

13. Prince (2004).

14. Crehan (2014).

15. Es interesante señalar el hecho inusual de que Dorothy Lee y Paulo Freire renunciaron o redujeron el tiempo de su nombramiento como profesores de la Universidad de Harvard. Esta decisión podría sugerir algo acerca de su sentido de libertad y autonomía para perseguir sus propios valores y praxis educativa. 